\title{
Comparação de métodos de medida indireta de massa de forragem em pasto de azevém anual (Lolium multiflorum Lam.)
}

\author{
A comparison of methods used to determine forage mass in italian ryegras pasture \\ (Lolium multiflorum Lam.)
}

\author{
Guilherme Fernandes Cauduro $^{1}$ Paulo César de Faccio Carvalho ${ }^{2}$ \\ Cristina Maria Pacheco Barbosa ${ }^{3}$ Robson Lunardi ${ }^{3}$ Alcides Pilau $^{3}$ \\ Fabiana Kellermann de Freitas ${ }^{3}$ Jamir Luís Silva da Silva ${ }^{4}$
}

RESUMO

Este trabalho foi realizado de maio a outubro de 2003, na Estação Experimental da Universidade Federal do Rio Grande do Sul (EEA-UFRGS), tendo como objetivo avaliar e comparar a precisão do disco medidor de forragem, do bastão graduado e do medidor de capacitância em predizer a massa de forragem de pastagens de azevém anual. O trabalho constou de dois experimentos: o experimento 1 no qual foram avaliados os três métodos numa situação em que o azevém apresentava-se em fase de estabelecimento num delineamento completamente casualizado, com 32 repetições e três tratamentos; e o experimento 2, no qual foi avaliada a massa de forragem pré e pós-pastejo pelo bastão graduado e pelo disco medidor em potreiros sob lotação rotacionada. $O$ delineamento experimental do experimento 2 foi o de blocos completamente casualizados, com quatro repetições; os tratamentos constaram de duas intensidades de pastejo (baixa e moderada) definidas por ofertas de forragem de 2,5 ou 5,0 vezes o potencial de consumo dos cordeiros que foram utilizados. No experimento 1, o bastão graduado apresentou o melhor coeficiente de determinação com a massa de forragem $\left(r^{2}=0,65\right)$. No experimento 2 , os melhores coeficientes de determinação foram obtidos quando o pasto, sob lotação rotacionada, foi avaliado com o bastão graduado.

Palavras-chave: bastão graduado, disco medidor de forragem, medidor de capacitância.

\section{ABSTRACT}

This work was conducted between May and October of 2003 at the Experimental Station of the Universidade Federal do Rio Grande do Sul (EEA-UFRGS) and was aimed at studing and comparing the efficiency of the rising plate meter, sward- stick and capacitance meter to predict the herbage mass in an italian ryegrass pasture. The work had two experiments: the experiment 1 where the three methods were evaluated when the pasture had free growth (establishment phase) in a completely randomized with 32 replicates and three treatments; the experiment 2 where pre and post grazing herbage mass was evaluated by the sward-stick and rising plate meter in paddocks under rotational grazing. The experimental design of experiment 2 was a completely randomized block with four replicates; the experimental treatments were two grazing intensities (low and moderate) defined by herbage allowances representing 2,5 and 5,0 times the potential intake of the lambs. In the experiment 1 the sward-stick showed the best determination coefficient $\left(r^{2}=0,65\right)$. In the experiment 2 the best determination coefficients were obtained when the pasture under rotational stocking was evaluated using the sward-stick.

Key words: sward-stick, rising plate meter, capacitance meter.

\section{INTRODUÇÃO}

O Brasil possui cerca de 160 milhões de cabeças de bovinos de corte, 1 milhão de bubalinos e 14 milhões de ovinos (ANUALPEC, 2004). Grande parte dessas espécies animais são criadas em pastagens. Para o manejo correto e eficiente dessas pastagens, o controle da oferta de forragem é um ponto decisivo; representa adequar-se uma disponibilidade de forragem a uma carga animal.

Para o cálculo da oferta de forragem, a massa de forragem do pasto deve ser mensurada. O

\footnotetext{
${ }^{1}$ Programa de Pós-graduação em Zootecnia, Universidade Federal do Rio Grande do Sul (UFRGS), Porto Alegre, RS, Brasil. E-mail: guicauduro@terra.com.br.

${ }^{2}$ Departamento de Plantas Forrageiras e Agrometeorologia, UFRGS. Av. Bento Gonçalves, 7712, 91501-970, Porto Alegre, RS, Brasil. E-mail: paulocfc@ufrgs.br. Autor para correspondência.

${ }^{3}$ Programa de Pós-graduação em Zootecnia, UFRGS, Porto Alegre, RS, Brasil.

${ }^{4}$ Faculdade de Engenharia Agronômica e Veterinária, Universidade Luterana do Brasil (ULBRA), Porto Alegre, RS, Brasil.
} 
monitoramento da variação da massa de forragem é uma das formas mais efetivas de gerar subsídios para diversos processos de gerenciamento e tomada de decisão sobre o manejo do pastejo ('T MANNETJE, 2000). A forma de maior confiabilidade é a estimação por meio de cortes da massa vegetal do pasto acima do solo. Essa forma é bastante trabalhosa e demorada, pois devem ser coletadas amostras suficientes para representar corretamente a área em estudo. Com a utilização de métodos indiretos de medição de massa de forragem, a avaliação torna-se menos demorada. No Brasil, a disponibilidade de técnicas para a realização desse tipo de estimativa é ainda incipiente, agravandose pelo grande número de espécies de plantas forrageiras utilizadas e pela carência de informações sobre a ecofisiologia das plantas (SILVA \& SBRISSIA, 2000). Dessa forma, existe interesse em que as técnicas de avaliação sejam melhoradas e possam otimizar os fatores tempo e mão-de-obra, desde que mantenham grau de confiabilidade satisfatório.

Entre os métodos indiretos de avaliação de massa de forragem estão o disco medidor de forragem (DS), o bastão graduado (BG) e o medidor de capacitância (MC). O DS consiste em um componente (quadrado ou redondo) de metal de um peso conhecido, o qual desliza ao longo de um eixo central. A altura da forragem acima do solo é mensurada pelo eixo central, que possui uma escala graduada, sendo medida por um contador, ou então as leituras são automaticamente armazenadas em um computador portátil. Este instrumento avalia a massa de forragem considerando sua altura e a densidade em função da pressão exercida pelo DS sobre o pasto.

O BG é constituído de uma haste metálica graduada e apresenta uma janela para que sejam realizadas as leituras de altura da planta por meio de um pequeno e transparente dispositivo de plástico (marcador móvel), o qual percorre a haste metálica. A base da haste é posicionada sobre o solo e o marcador deve ser elevado à parte superior da haste. Logo após, o marcador móvel deve ser deslizado e, ao primeiro toque na folha da planta, realiza-se a leitura da escala graduada. A altura pode ser medida em intervalos de $0,5 \mathrm{~cm}$. O pasto, diferentemente do DS, não é comprimido. Já o funcionamento do MC é baseado em um sinal produzido por um oscilador de circuito elétrico, sendo os sinais captados pelo sensor de capacitância. O aparelho consiste em uma sonda leve, onde os dados são automaticamente armazenados para serem analisados em computador, ou então o instrumento pode ser calibrado com uma equação. Deste modo, as leituras em kg de matéria seca são automaticamente realizadas e apresentadas ao operador. Deve ser feita a calibração do aparelho sempre antes de cada amostragem no pasto em razão de os valores das leituras dependerem do tipo de pasto e de sua umidade (' $\mathrm{T}$ MANNETJE, 2000).

O objetivo deste estudo foi o de avaliar e comparar três métodos de medida indireta de massa de forragem. Foram utilizados o DS, o BG e o MC em um pasto de azevém em situação de crescimento livre no primeiro experimento. Em um segundo experimento, o DS e o BG foram avaliados quando o pasto apresentavase sob lotação rotacionada, estudando-se as massas de forragem de pré e pós-pastejo em duas intensidades de pastejo.

\section{MATERIALEMÉTODOS}

O experimento foi conduzido na Estação Experimental Agronômica da UFRGS. As coordenadas geográficas são 3005’22”' S e 51³9’08” W. O clima da região é subtropical úmido com verões quentes, tipo fundamental "Cfa” da classificação climática de Köppen (MORENO, 1961). A temperatura média anual é de $19,3^{\circ} \mathrm{C}$. Há formação ocasional de geadas no período de maio a setembro, com maior incidência nos meses de junho, julho e agosto. A precipitação média anual situa-se ao redor de $1440 \mathrm{~mm}$. Os dados meteorológicos durante o período experimental foram coletados em uma Estação Meteorológica, a cerca de $1000 \mathrm{~m}$ da área experimental (Tabela 1).

O experimento foi instalado em um [ARGISSOLO VERMELHO] Distrófico típico (EMBRAPA CNPS, 1999), sendo o terreno levemente ondulado, sem limitações para cultivos anuais. Para a semeadura do pasto de azevém, foi necessária a dessecação da área experimental (13/03/2003) com a utilização de herbicida de princípio ativo Glifosate na dosagem de $5 \mathrm{~L} \mathrm{ha} \mathrm{h}^{-1}$. Após isso, foi necessária a roçada da vegetação.

Para determinar a fertilidade do solo, foram realizadas amostragens na profundidade de $0-10 \mathrm{~cm}$. A análise de solo revelou: argila (\%), 19,0; $\mathrm{pH}\left(\mathrm{H}_{2} \mathrm{O}\right)$, 5,3; M.O.(\%), 2,9; P (ppm), 13,5; K (ppm) 135,0; Al troc. $\left(\mathrm{cmol} \mathrm{L}_{\mathrm{c}}^{-1}\right), 0,3$; Ca trocável $\left(\mathrm{cmol} \mathrm{L}_{\mathrm{c}}^{-1}\right), 2,1 ; \mathrm{Mg}\left(\mathrm{cmol}_{\mathrm{c}}\right.$ $\mathrm{L}^{-1}$ ), 1,2; saturação por bases da CTC (\%), 54,2.

A adubação e a calagem utilizadas seguiram a Recomendação de Adubação e Calagem para os Estados do RS e SC (SBCS, 1989). Foi aplicada a quantidade de 1 ton ha-1 de calcário (16/04/2003) e 200kg $\mathrm{ha}^{-1}$ de adubo na fórmula 5-20-20. A semeadura do pasto (21/04/03) foi feita com a utilização de máquina semeadora-adubadora tratorizada de plantio direto, com espaçamento entre linhas de $17 \mathrm{~cm}$. A densidade de semeadura foi de $32 \mathrm{~kg} \mathrm{ha}^{-1}$ de sementes de azevém anual (Lolium multiflorum Lam). 
Tabela 1 - Radiação solar (Rs, em cal.cm ${ }^{-2} \cdot \mathrm{dia}^{-1}$ ), temperaturas médias mensais do ar (média, máxima e mínima, em ${ }^{\circ} \mathrm{C}$ ) e precipitação pluviométrica média (Prec., em mm), observadas ao longo do período experimental. EEA/UFRGS, 2003.

\begin{tabular}{|c|c|c|c|c|c|}
\hline \multirow[t]{2}{*}{ Meses } & Rs & \multicolumn{3}{|c|}{ Temperatura $\left({ }^{\circ} \mathrm{C}\right)$} & \multirow{2}{*}{$\begin{array}{c}\text { Prec. } \\
(\mathrm{mm})\end{array}$} \\
\hline & $\left(\mathrm{cal} . \mathrm{cm}^{-2} \cdot \mathrm{dia}^{-1}\right)$ & Média & Máxima & Mínima & \\
\hline março & 399,90 & 22,88 & 29,12 & 17,94 & 91,48 \\
\hline abril & 299,03 & 18,39 & 24,86 & 12,80 & 115,70 \\
\hline maio & 248,84 & 15,74 & 22,37 & 10,18 & 41,00 \\
\hline junho & 144,71 & 15,62 & 20,14 & 11,65 & 161,24 \\
\hline julho & 185,50 & 12,93 & 19,08 & 7,77 & 138,67 \\
\hline agosto & 265,61 & 12,53 & 19,64 & 6,17 & 60,67 \\
\hline setembro & 313,59 & 14,90 & 21,92 & 8,63 & 68,69 \\
\hline outubro & 400,12 & 19,00 & 25,50 & 13,41 & 277,35 \\
\hline
\end{tabular}

Em relação à adubação nitrogenada em cobertura, foram utilizados na área experimental $150 \mathrm{~kg}$ N.ha-1 sob forma de uréia, conforme FREITAS (2003), que determinou essa quantidade de nitrogênio como a mais indicada para o uso de azevém em pastejo com ovinos. A adubação nitrogenada foi fracionada e aplicada em dois momentos: metade da dose aplicada na emissão da 4⿳亠丷a folha de azevém (24/05/03 - em razão de ser o início do perfilhamento e o período de maior demanda de compostos nitrogenados pela planta) e o restante no início do período de primavera (5/09/03).

A área total do experimento foi de aproximadamente 6ha. Desse total, 1,7ha foram destinados aos animais reguladores e 4,3ha foram utilizados para as unidades experimentais (UE). O tamanho médio das UE foi, portanto, de 0,26ha. A avaliação dos métodos indiretos de medição de massa de forragem foi realizada em dois experimentos, diferenciados pelos métodos que foram comparados e pela existência ou não da ação do pastejo.

No Experimento 1, com o pasto em crescimento livre na fase de estabelecimento, foi realizada a avaliação com o MC, o BG e o DS no dia 25/ 06/2003 num delineamento completamente casualizado, com 32 repetições. O MC utilizado foi o modelo Grassmaster II, da marca Speedrite; o DS apresentava uma área de $0,1 \mathrm{~m}^{2}$ e o BG apresentava uma escala para medição de altura da planta de até $50 \mathrm{~cm}$. Na área experimental, foram realizados 32 cortes destrutivos da vegetação em pontos aleatórios. Em cada ponto foi fixado um quadro de metal de área de $0,25 \mathrm{~m}^{2}$ e, na área delimitada pelo quadro, foram realizadas, antes do corte da vegetação, cinco amostragens com o MC, cinco medidas com o BG e uma medida com o DS.

A avaliação com o MC consistiu no posicionamento do aparelho na vegetação e, automaticamente, o aparelho indicava no seu visor de leitura a média do valor de massa de forragem (em kg ha-1 de MS) dos cinco pontos amostrados aleatoriamente na área delimitada pelo quadro. Para a medição da altura do pasto com o BG, foi necessário o posicionamento do bastão verticalmente na vegetação, onde a base de sua haste metálica ficava em contato com o solo. Posteriormente o dispositivo plástico (marcador móvel) era deslizado até a parte superior da haste metálica do equipamento. Logo após, o marcador era então deslizado em direção ao solo e, ao primeiro toque em uma lâmina foliar de uma planta de azevém, o dispositivo não era mais movimentado, realizando-se então a leitura da altura da planta na escala graduada (indicada pelo marcador) presente na haste do bastão graduado (conforme HODGSON, 1990). Esse procedimento era repetido cinco vezes em pontos aleatórios do quadro metálico.

O procedimento seguinte consistia na avaliação da vegetação limitada pelo quadro metálico com o uso do DS. O processo de medição se dava com o posicionamento do DS verticalmente na vegetação (nesse momento era efetuada a leitura do contador do aparelho); após isso, o eixo central metálico do equipamento era empurrado em direção ao solo. No momento em que a base metálica do eixo central entrasse em contato com o solo, esta era puxada de volta a sua posição original. Após esse procedimento, a leitura do contador era novamente efetuada. $\mathrm{O}$ valor mensurado do aparelho era calculado subtraindo-se a leitura final do contador da leitura inicial.

Por último, a forragem limitada pelo quadro metálico era cortada em nível do solo com a utilização de tosquiadeiras elétricas acopladas a um gerador, tomando-se o cuidado de não contaminar a amostra da vegetação. Após isso, quantificava-se a matéria seca das amostras.

Ciência Rural, v.36, n.5, set-out, 2006. 
Posteriormente, para validação dos métodos, os pares de dados de leitura de altura da planta x massa de forragem, leitura de matéria seca do medidor de capacitância x massa de forragem, leitura do disco $\mathrm{x}$ massa de forragem foram tabulados e efetuada uma análise de regressão até terceira ordem (PROC REG; pacote estatístico SAS versão 6.0, 1996), com nível de $5 \%$ de probabilidade. No momento em que a equação gerada pelo programa estatístico apresentasse significância, era adotada a equação que apresentasse o coeficiente de determinação mais elevado.

O experimento 2 consistiu na avaliação da mesma vegetação, mas submetida a pastejo por meio de lotação rotacionada; foi comparada a eficiência do DS e do BG em predizer a massa de forragem existente em pré-pastejo e em pós-pastejo. O tratamento experimental constou de duas intensidades de pastejo (IP) definidas por ofertas de forragem de 2,5 e 5,0 vezes o consumo dos ovinos (IP moderada e baixa, respectivamente), sendo o potencial de consumo de cordeiros, cruzas Texel e Ile de France (idade média inicial de nove meses), de $4 \%$ do peso vivo (PV) (NRC, 1985). Foi utilizado número variável de animais reguladores para ajuste da oferta de forragem pela técnica “put-and-take” (MOTT \& LUCAS, 1952).

O pastejo se iniciou em 12/07/2003 e o ciclo de pastejo compreendeu períodos de ocupação de dois dias e período de descanso variável segundo a duração de vida da folha do azevém (de 22 a 35 dias). O delineamento experimental foi o de blocos casualizados, com quatro repetições. A avaliação do pasto ocorreu em três datas: 11/9, 3/10 e 7/11/2003, conforme procedimento já descrito anteriormente. Foram amostrados quatro pontos aleatoriamente por unidade experimental; dois pontos em faixas de pré-pastejo e dois pontos em faixas de pós-pastejo. Em cada ponto eram realizadas cinco medições de altura da planta com o BG e uma amostragem com o DS. O procedimento de corte da forragem, tabulação dos pares de dados de leitura do DS x massa de forragem e a análise estatística foram os mesmos adotados no Experimento 1.

\section{RESULTADOS EDISCUSSÃO}

Os resultados encontrados no experimento 1 estão na tabela 2. O instrumento que obteve correlação aceitável foi o BG, sendo o instrumento preditor com maior eficiência para determinação de massa de forragem em pastos de azevém, apresentando $\mathrm{r}^{2}=0,655$. BANDINELLI et al. (2003), avaliando a eficiência do BG e do DS em um pasto consorciado de aveia e azevém, também demonstraram valores superiores para o primeiro método $\left(\mathrm{r}^{2}=0,506\right.$ para o $\mathrm{BG}$ e $\mathrm{r}^{2}=0,364$ para o DS). Os autores reportaram que uma possível causa da baixa acurácia do DS foi a alteração na estrutura do pasto em função de uma elevação dos colmos no estrato superior. Essa também pode ser uma razão da baixa precisão encontrada do DS na avaliação realizada nesse experimento. No momento da avaliação dos métodos, algumas plantas de azevém apresentavam mudança de estrutura (elevação de colmos), com valores de massa de forragem seca superiores a $3.000 \mathrm{~kg} \mathrm{ha}^{-1}$. Já HARMONEY et al. (1997), avaliando pasto de Festuca arundinacea Schreb encontraram valores de $\mathrm{r}^{2}=0,08 \mathrm{e}$ $\mathrm{r}^{2}=0,85$ para o BG e o DS, respectivamente.

$\mathrm{O}$ menor $\mathrm{r}^{2}$ foi encontrado no $\mathrm{MC}$, apresentando $r^{2}=0,14$. MURPHY et al. (1995), testando um MC em pasto de P. pratensis L. e T. repens L., encontraram $r^{2}=0,42$, valor este bem superior ao encontrado neste experimento. COLEMAN \& FORBES (1998) determinaram valores de $\mathrm{r}^{2}$ para o MC em um pasto de Bothriocloa spp. durante dois anos. No primeiro ano, os valores encontrados foram de $\mathrm{r}^{2}=0,54$ a $\mathrm{r}^{2}=0,77$; para o segundo ano, de $\mathrm{r}^{2}=0,27 \mathrm{a} \mathrm{r}^{2}=0,63$.

SANDERSON et al. (2001) avaliaram pastagens consorciadas de gramíneas de estação fria (F. arundinacea Schreb, P. pratensis L., T. repens L., L. perenne L., D. glomerata L.) com o MC, o DS e o BG. A avaliação do pasto para determinação indireta de matéria seca com os métodos deu-se no momento em que o pasto apresentava em torno de $2.500 \mathrm{~kg} \mathrm{ha}^{-1} \mathrm{de}$ MS (valor próximo à massa de forragem verificada neste experimento). Os valores encontrados pelos autores foram de $\mathrm{r}^{2}=0,19$ (MC), $\mathrm{r}^{2}=0,31$ (DS) e $\mathrm{r}^{2}=0,16$ (BG). Assim como neste experimento, esses dados demonstram que os métodos não foram precisos para estimar a massa de forragem do pasto.

Tabela 2 - Métodos preditores de massa de forragem (MC - medidor de capacitância; DS - disco medidor de forragem; BG - bastão graduado), coeficiente de correlação de Pearson (r), probabilidade (Pr), coeficiente de determinação $\left(r^{2}\right)$, probabilidade (Pr), equação de regressão e coeficiente de variação (CV).

\begin{tabular}{lccccll}
\hline Método & $\mathrm{r}$ & $\operatorname{Pr}$ & $\mathrm{r}^{2}$ & $\operatorname{Pr}$ & Equação & CV (\%) \\
\hline MC & 0,386 & 0,0289 & 0,149 & 0,028 & $\mathrm{y}=138,4+0,519 \mathrm{x}$ & 31,54 \\
DS & 0,454 & 0,0089 & 0,206 & 0,0089 & $\mathrm{y}=2,32+0,009 \mathrm{x}$ & 25,71 \\
BG & 0,693 & 0,0001 & 0,655 & 0,0001 & $\mathrm{y}=123,969+0,081 \mathrm{x}-0,00001 \mathrm{x}^{2}$ & 10,31 \\
\hline
\end{tabular}


SILVA \& DA CUNHA (2003), avaliando o DS e o BG em três espécies de Cynodon spp. no decorrer de um ano, obtiveram valores de $\mathrm{r}^{2}=0,49$ a $\mathrm{r}^{2}=0,86$ para o DS e de $\mathrm{r}^{2}=0,4$ a $\mathrm{r}^{2}=0,85$ para o BG. Os valores de $r^{2}$ variam em função da época do ano em que foi realizada a avaliação. De forma geral, os valores encontrados pelos autores não atingiram o grau de precisão determinado por THOMSON (1986) (coeficiente de determinação mínimo de 0,75) para serem utilizados como métodos preditores, à exceção de quando os equipamentos foram utilizados no período de verão (janeiro a março). THOMSON (1986) afirmou que valores de coeficiente de determinação abaixo de 0,75 são insatisfatórios.

Os valores dos $\mathrm{r}^{2}$ do experimento 1 foram baixos, indicando que parte significativa da variação da massa de forragem não pôde ser explicada pela resposta do MC e pela variação em altura e densidade da forragem (DS e BG). Os valores de $\mathrm{r}^{2}$ encontrados entre a predição de massa de forragem pelos métodos e a massa de forragem existente no pasto foram abaixo de 0,75 (preconizado por THOMSON, 1986). Dessa forma, os modelos matemáticos (equações geradas de cada instrumento) para predição de massa de forragem testadas nas circunstâncias desse experimento tornamse de emprego limitado.

Em grande parte dos resultados dos experimentos apresentados, os métodos preditores não atingiram os níveis de confiabilidade requeridos por THOMSON (1986). Uma questão que deve ser considerada é o fato de que, no momento da medição da massa de forragem pelos métodos, fatores de alto grau de variabilidade, tais como a umidade do ar e a umidade da forragem, a superfície do solo muito irregular, o pisoteio e o amassamento do pasto pelos animais são determinantes na avaliação (AIKEN \& BRANSBY, 1992). Uma característica particular das condições desse experimento foi o estabelecimento do pasto em sistema de plantio direto, o qual se caracteriza pela manutenção de elevada quantidade de material morto na forma de palha acima do solo. É possível que este tipo de situação tenha sido responsável pelo baixo valor preditivo dos métodos, em particular do MC, cuja natureza de funcionamento estaria mais suscetível a erros com a existência da referida camada de palha.

$\mathrm{O}$ aparelho preditor de massa de forragem mais confiável (BG) foi o que apresentou um coeficiente de variação (CV) mais baixo (10,31\%). Esse valor de CV implica em amplitudes de diferenças de massa de forragem aceitáveis tanto para situações de pesquisa, a qual necessita de certo rigor, como em manejo do pasto para produtores e para o planejamento estratégico de disponibilidade de forragem. Essa situação pode ser exemplificada por um pasto que apresente $1.000 \mathrm{~kg}$ ha $^{-1}$ de MS. Nesse caso, a massa de forragem medida pelo BG teria uma variação de $896,9 \mathrm{~kg} \cdot \mathrm{ha}^{-1}$ de MS a $1.103,1 \mathrm{~kg} \mathrm{ha}^{-1}$ de MS. Um fato relevante para os baixos valores de predição dos métodos avaliados no experimento 1 pode ter sido o reduzido número de amostragens realizadas para determinar as curvas de calibração e correlação.

Para a avaliação do experimento 2, é necessário destacar que houve diferença entre as ofertas de forragem $(\mathrm{ORF})(\mathrm{P}<0,05)$, determinando então diferenças entre as intensidades de pastejo adotadas. Os valores de ORF para as intensidades de pastejo (IP) baixa e moderada foram, respectivamente, de 18,3 e 9,6\% PV, valores próximos aos preconizados por PONTES et al. (2004), que determinaram esse valores como, respectivamente, quantidade de pasto superior e limitante ao consumo dos animais.

Os resultados do experimento 2 estão descritos na tabela 3. Segundo os resultados, a correlação entre os aparelhos e a massa de forragem foi maior quando avaliadas as faixas pós-pastejo. Conseqüentemente, as faixas de pastejo finais apresentavam uma uniformidade, diminuindo, então, a variabilidade da vegetação que interfere nas respostas do aparelho preditor de massa de forragem. Já o BG apresentou maior correlação entre as leituras do instrumento e a massa de forragem presente no pasto no tratamento IP moderada em faixa pós-pastejo. É possível que isso tenha ocorrido em função de o pasto, nesse tratamento, não encontrar-se em estádio reprodutivo (fato que ocorreu nos tratamentos de IP baixa); dessa forma, seu perfil apresentava grande proporção de folhas. Deve-se considerar que este instrumento determina a massa de forragem do pasto levando em conta a altura das folhas da planta.

SORIANO (1998), avaliando o DS em pastagens de $\boldsymbol{P}$. pratensis, $\boldsymbol{T}$. repens e $\boldsymbol{D}$. glomerata (espécie preponderante no pasto), manejadas em lotação rotacionada, determinou uma correlação de $r=$ 0,73 entre as respostas do instrumento e a massa de forragem. CASTLE (1976) determinou valores de correlação entre r = 0,39 e r = 0,62 em pasto de Festuca arundinacea Schreb manejada rotacionalmente. MARTIN et al. (2005), avaliando a eficiência do DS e do BG, encontraram valores de $\mathrm{r}^{2}$ de 0,35 a 0,83 para o DS e de 0,11 a 0,8 para o BG em faixas de pré-pastejo e $\mathrm{r}^{2}$ de 0,07 a 0,81 e de 0,06 a 0,86 em faixas de pós-pastejo em pasto consorciado de $\boldsymbol{P}$. pratense L., F. pratensis Huds, L. perenne e T. repens L.

O nível de significância das equações geradas pelos tratamentos foi elevado (Prob $>$ F), à 
Tabela 3 - Avaliação do disco medidor de forragem e do bastão graduado nos tratamentos (Trat.) intensidade de pastejo moderada e faixa pré-pastejo (MPRP), intensidade de pastejo moderada e faixa pós-pastejo (MPOP), intensidade de pastejo baixa e faixa prépastejo (BPRP), intensidade de pastejo baixa e faixa pós-pastejo (BPOP), coeficiente de correlação de Pearson (r), probabilidade $(\mathrm{Pr})$, coeficiente de determinação $\left(\mathrm{r}^{2}\right)$, probabilidade (Pr), equação de regressão e coeficiente de variação $(\mathrm{CV})$.

\begin{tabular}{|c|c|c|c|c|c|c|}
\hline \multicolumn{7}{|c|}{ Disco medidor de forragem } \\
\hline Trat. & $\mathrm{r}$ & $\operatorname{Pr}$ & $r^{2}$ & $\operatorname{Pr}$ & Equação & CV (\%) \\
\hline MPRP & $-0,248$ & 0,241 & - & - & - & - \\
\hline MPOP & 0,691 & 0,0002 & 0,478 & 0,0002 & $y=3,295+0,0063 x$ & 46,56 \\
\hline BPRP & 0,494 & 0,015 & 0,325 & 0,0159 & $y=46,892-0,01 x+0,000001 x^{2}$ & 36,21 \\
\hline BPOP & 0,65 & 0,0006 & 0,423 & 0,0006 & $y=15,717+0,0034 x$ & 15,98 \\
\hline & \multicolumn{6}{|c|}{ Bastão graduado } \\
\hline Trat. & $\mathrm{r}$ & $\operatorname{Pr}$ & $\mathrm{r}^{2}$ & $\operatorname{Pr}$ & Equação & CV (\%) \\
\hline MPRP & 0,731 & 0,0001 & 0,367 & 0,0017 & $y=6,588+0,002 x$ & 23,07 \\
\hline MPOP & 0,136 & 0,0002 & 0,559 & 0,0002 & $y=1,45+0,01 x-0,0000009 x^{2}$ & 12,80 \\
\hline BPRP & 0,564 & 0,523 & - & - & - & - \\
\hline BPOP & 0,606 & 0,0001 & 0,535 & 0,0001 & $y=5,382+0,004 x$ & 28,11 \\
\hline
\end{tabular}

exceção dos tratamentos IP moderada e faixa prépastejo para o DS e IP baixa e faixa pré-pastejo para o BG. Entretanto, os valores dos $\mathrm{r}^{2}$ foram baixos, indicando que parte significativa da variação de massa de forragem não pôde ser explicada pela resposta dos métodos preditores de massa de forragem.

Por último, para validação dos métodos preditores indiretos de massa de forragem, são necessárias diversas amostragens a fim de que esses métodos sejam corretamente calibrados (leitura do aparelho x quantidade de matéria seca presente no pasto) e apresentem valores de coeficiente de determinação aceitáveis. Dessa forma, os métodos preditores de massa de forragem podem se tornar ferramentas importantes para o monitoramento e o planejamento da disponibilidade de forragem tanto para situações de pesquisa como para manejo dos sistemas de produção animal que utilizam pastagens.

\section{CONCLUSÃO}

Quando o pasto se apresenta em crescimento livre, os métodos avaliados não são suficientemente precisos para serem utilizados como preditores da massa de forragem. $\mathrm{O}$ instrumento que mais se aproxima de um coeficiente de determinação aceitável é o bastão graduado.

A partir da ação do animal em pastejo, o bastão graduado é o método mais indicado para predizer a massa de forragem em lotação rotacionada.

\section{AGRADECIMENTOS}

Os autores agradecem aos integrantes do Grupo de Pesquisa em Ecologia do Pastejo da Universidade Federal do
Rio Grande do Sul (UFRGS); à Empresa Agropecuária Cerro Coroado, à Coordenação de Aperfeiçoamento de Pessoal de Nível Superior (CAPES), pela concessão de bolsa ao pesquisador Cauduro; ao Conselho Nacional de Desenvolvimento Científico e Tecnológico (CNPq), pela concessão de bolsa aos pesquisadores Barbosa e Freitas.

\section{REFERÊNCIAS}

AIKEN, G.E.; BRANSBY, D.I. Observer variability for disk meter measurements of forage mass. Agronomy Journal, Madison, v.84, n.4, p.603-605, 1992.

ANUALPEC 2004: Anuário da pecuária brasileira. São Paulo:FNP Consultoria/GERDAU, 2004. 376p.

BANDINELLI, D.G. et al. Comparação de métodos para estimativa da massa de forragem em gramíneas de estação fria, Santa Maria, RS, 2003. In: REUNIÃO ANUAL DA SOCIEDADE BRASILEIRA DE ZOOTECNIA, 40., 2003, santa Maria, RS. Anais... Santa Maria: Sociedade Brasileira de Zootecnia, 2003. (CD-Rom).

CASTLE, M.E. A simple disc instrument for estimating herbage yield. Journal of British Grassland Society, Oxford, v.31, n.2, p.37-40, 1976.

COLEMAN, S.W.; FORBES, T.D.A. Herbage characteristics and performance of steers grazing old world bluestem. Journal of Range Management, Denver, v.51, n.4 p.399-407, 1998.

EMBRAPA, CENTRO NACIONAL DE PESQUISA DE SOLOS. Sistema Brasileiro de Classificação de Solos. Brasília: Embrapa Produção de Informação; Rio de Janeiro: Embrapa Solos, 1999. 412p.

FREITAS, T.M.S. de. Dinâmica da produção de forragem, comportamento ingestivo e produção de ovelhas Ile de France em pastagem de azevém anual (Lolium multiflorum Lam.) em resposta a doses de nitrogênio. 2003. 152f. Dissertação (Mestrado em Zootecnia) - Curso de Pós-graduação em Zootecnia, Universidade Federal do Rio Grande do Sul. 
HARMONEY, K.R. et al. Determination of pasture mass using four indirect methods. Agronomy Journal, Madison, v.89, n.2, p.665-672, 1997.

HODGSON, J. Grazing management: science into practice. New York: John Wiley \& Sons, 1990. 203p.

MARTIN, R.C. et al. A comparison of methods used to determine biomass on naturalized swards. Journal of Agronomy \& Crop Science, Berlin, v.191, n.2, p.152-160, 2005.

MORENO, J.A. Clima do Rio Grande do Sul. Porto Alegre: Governo do Estado do Rio Grande do Sul - Secretaria da Agricultura, 1961. 41p.

MOTT, G.O.; LUCAS, H.L. The design, conduct, and interpretation of grazing trials on cultivated and improved pastures. In: INTERNATIONAL GRASSLAND CONGRESS, 6., 1952, Pennsylvania. Proceedings... Pennsylvania: Pensylvania State College, 1952. p.1380-1385.

NATIONAL RESEARCH COUNCIL - NRC. Nutrient requirement of sheep. 6.ed. Washington: National Academy of Science, 1985. 99p.

MURPHY, W.M. et al. A comparison of quadrate, capacitance meter, HFRO sward stick and rising plate meter for estimating herbage mass in a smooth-stalked meadowgrass-dominant white clover sward. Grass and Forage Science, Oxford, v.50, n.3, p.452-455, 1995.

PONTES, L.S. et al. Variáveis morfogênicas e estruturais de azevém anual (Lolium multiflorum Lam.) manejado em diferentes alturas. Revista Brasileira de Zootecnia, Viçosa, v.33, n.3, p.529-537, 2004.
SANDERSON, M.A. et al. Estimating forage mass with a commercial capacitance meter, rising plate meter and pasture ruler. Agronomy Journal, Madison, v.93, n.6, p.1281-1286, 2001.

SAS INSTITUTE. SAS/STAT user's guide: statistics. Version 6. Cary, NC, 1996. V.2. 943p.

SBCS, Núcleo Regional Sul. Recomendações de adubação e calagem para os estados do Rio Grande do Sul e Santa Catarina. 2.ed. Passo Fundo, RS, 1989. 128p.

SORIANO, F.D. Grazing and feeding management in lactating dairy cows, 1998. Capturado em 06 mar. 2005. Online. Disponível na internet: http://scholar.lib.vt.edu/theses/ available/etd-71198-21915/unrestricted/thesisfelix.pdf

SILVA, S.C. da.; SBRISSIA, A.F. A planta forrageira no sistema de produção, Piracicaba, SP, 2000. In: SIMPÓSIO SOBRE O MANEJO DA PASTAGEM, 17., 2000, Piracicaba, SP. Anais... Piracicaba: Fundação de Estudos Agrários Luiz de Queiroz, 2000. p.3-21.

SILVA, S.C. da; CUNHA, W.F. da. Métodos indiretos para estimar a massa de forragem em pastos de Cynodon spp. Pesquisa Agropecuária Brasileira, Brasília, v.38, n.8, p.981-989, 2003.

'T MANNETJE, L. Measuring biomass of grassland vegetation. In: 'T MANNETJE, L.; JONES, R.M. (Ed). Field and laboratory methods for grassland and animal production research. Wallingford: CABI Publishing/CAB International, 2000. Cap.7, p.151-177.

THOMSON, N.A. Techniques available for assessing pasture. Dairy Farming Annual, Palmerston North, v.38, n.2, p.113121, 1986 